J. Genet. Vol. 69, No. 3, December 1990, pp. 127-139. (C) Printed in India.

\title{
Transposable element-medicated evolution of sex: A population genetic model
}

\author{
N. V. JOSHI \\ Centre for Ecological Sciences, Indian Institute of Science, Bangalore 560012, India \\ MS received 27 July 1990
}

\begin{abstract}
For a population made up of individuals capable of sexual as well as asexual modes of reproduction, conditions for the spread of a transposable element are explored using a one-locus, two-haplotype model. The analysis is then extended to include the possibility that the transposable element can modulate the probability of sexual reproduction, thus casting Hickey's (1982, Genetics 101: 519-531) suggestion in a population genetics framework. The model explicitly includes the cost of sexual reproduction, fitness disadvantage to the transposable element, probability of transposition, and the predisposition for sexual reproduction in the presence and absence of the transposable element. The model predicts several kinds of outcome, including initial frequency dependence and stable polymorphism. More importantly, it is seen that for a wide range of parameter values, the transposable element can go to fixation. Therefore it is able to convert the population from a predominantly asexual to a predominantly sexual mode of reproduction. Viewed in conjunction with recent results implicating short stretches of apparently non-coding DNA in sex determination (McCoubrey et al. 1988, Science 242: 1146-1151), the model hints at the important role this mechanism could have played in the evolution of sexuality.
\end{abstract}

Keywords. Selfish DNA; transposable clements; evolution of sex.

\section{Introduction}

The existence of mobile genetic elements in eucaryotic organisms has been known for many years (see Shapiro 1983, Berg and Howe 1989). Many conjectures have been made about their possible biological function. However, it was pointed out by Orgel and Crick (1980) and Doolittle and Sapienza (1980) that the ability of these mobile genetic elements to transpose (replicate themselves within as well as between genomes) was sufficient for them to spread under the action of natural selection; no functional role was necessary (see Doolittle 1982 for a critical review). Hickey (1982) described an explicit quantitative model for this process and obtained conditions under which transposable elements (TEs) can spread in a population despite having a fitness disadvantage. More sophisticated deterministic and stochastic models describing the equilibrium copy number of TEs have been described (Ohta and Kimura 1981; Ohta 1981, 1983; Charlesworth and Charlesworth 1983; Langley et al. 1983) while Nanjundiah (1985), using one-locus, two-haplotype models, demonstrated the possibility of stable polymorphisms with regard to the presence or absence of a TE. The population genetics of TEs continues to be a flourishing area of investigation (see Charlesworth and Langley 1989, for a recent review). 
Hickey (1982) perceptively pointed out that sexuality (more precisely, outbreeding) was essential for the spread of TEs and made the intriguing suggestion that the evolution of sexuality itself may have been facilitated by TEs. The evolution of sex has been one of the most fascinating and challenging problems in biology for more than a century and has led to a variety of imaginative hypotheses (Williams 1975; Maynard Smith 1978; Bell 1982; Stearns 1985; Michod and Levin 1988). However, Hickey's suggestion does not seem to have received widespread attention (see e.g., Stearns 1985, 1990; Bremmermann 1985; Charlesworth 1987, 1989). Population dynamics models based on this idea (contagion models) have been explored by Rose and coworkers (Rose 1983; Tremblay and Rose 1985; Krieber and Rose 1986) as well as by Zinder (1985). They have demonstrated that invasion of an asexual population by a sexual one is possible under a wide range of conditions. However, the conditions required for the sexual type to go to fixation were found to be rather restrictive. Hickey and Rose (1988), in addition to the summary of these investigations, describe considerable supportive evidence in favour of the model. Curiously, an explicit population genetic formulation of this phenomenon has not been investigated in detail. Such a model is explored here.

In this paper I examine the dynamics of TEs which are able to affect the mode of sexuality in the host population. In particular, I consider a population in which a fixed proportion of individuals reproduces sexually and the rest asexually. The presence of a TE is assumed to control this proportion. It is seen that under a suitable range of parameter values, the TE can go to fixation and can convert the population to an exclusively sexual mode of reproduction.

\section{The model}

To keep the inherent features of the model as simple as possible, an infinite, panmictic population of haploid organisms with discrete non-overlapping generations is assumed. A one-locus, two-haplotype model for transposable elements is considered (Nanjundiah 1985) such that $T$ indicates the presence and $t$, the absence of a transposable element.

The model is schematically illustrated in figure 1, and definitions of the symbols used in the text are given in table 1 . The population is assumed to have two modes of reproduction with only a fraction of individuals able to reproduce sexually. The magnitude of this fraction is assumed to be determined by the presence or absence of $T$. Thus of the individuals with $t$ haplotype, a fraction $X$ reproduces sexually and of those carrying $T$, a fraction $Y$ reproduces sexually.

The sexually reproducing individuals intermingle and mate randomly to form diploid zygotes. The cost of sexual reproduction is modelled by the parameter $Z$ such that the relative fitness of sexually reproducing individuals is $(1-Z)$. This fitness handicap is assumed to be made manifest in the (transient) diploid stage fomed by the union of two sexually reproducing haplotypes.

In $T t$ zygotes, transposition is assumed to occur with probability $F$ so as to convert it to $T T$. It is this property of transposition which justifies the term 'selfish' or 'parasitic' DNA and enables $T$ haplotypes to spread in the population. Though such elements are known to be present in multiple copies in a genome, analysis is restricted in the present model to the presence of a single copy; additional copies are unlikely 


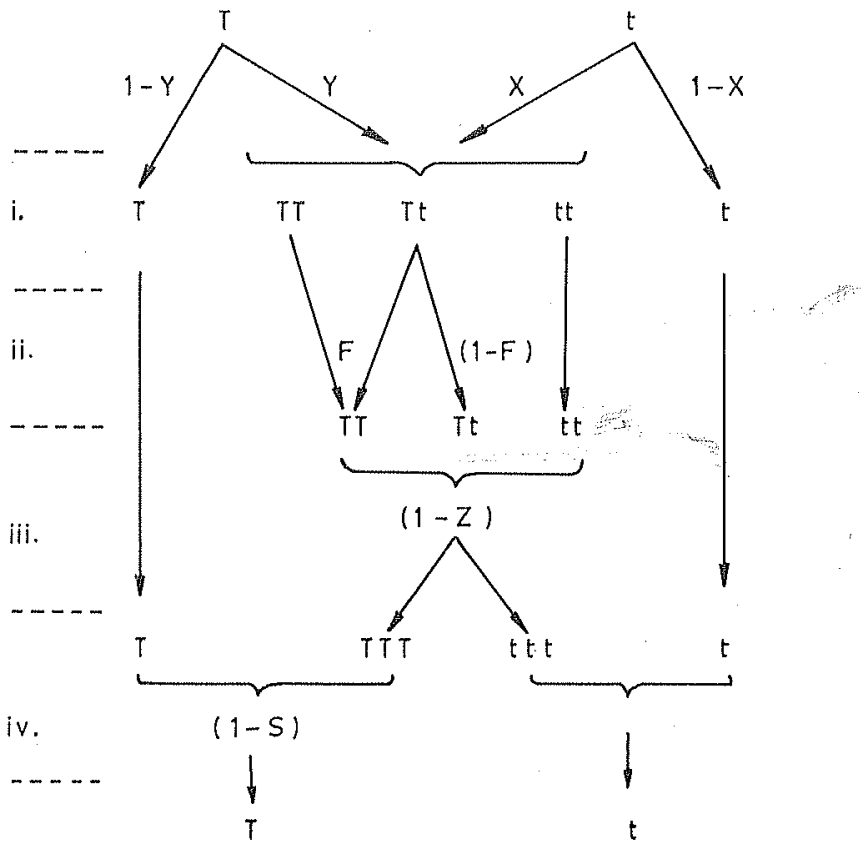

Figure 1. Schematic representation of the model, indicating the different processes responsible for bringing about the change in the frequency of the transposable element $T$ from one generation to the next. The right and left columns depict the asexual pathway for the $T$ and the $t$ haplotypes respectively, while the central column shows the fate of the zygotes. The four stages indicated in the figure are: (i) Zygote formation by random mating in the mating pool formed by the sexually reproducing fractions of $T$ and $t$; (ii) transposition in a fraction $F$ of the $T t$ zygotes, converting them into $T T$; (iii) sexual reproduction, with associated cost $(1-Z)$ relative to asexual reproduction; (iv) survival to adulthood, $(1-S)$ for $T$ haplotypes relative to $t$.

to change the qualitative features of the model. Following Hickey (1982), the $T$ haplotypes are assumed to have a further fitness disadvantage $S$, such that the relative probability of survival to adulthood is $(1-S)$. Several features of the basic framework of the model are very similar to the model proposed by Hoekstra (1990) for the evolution of male-female dimorphism.

Table 1. Symbols used and their definitions.

$T \quad$ Haplotype with the transposable element

$t \quad$ Haplotype without the transposable element

$F \quad$ Probability of transposition (conversion of $T t$ to $T T$ )

$S \quad$ Fitness disadvantage to $T$ haplotype, such that the probability of survival to adulthood is $(1-S)$

$X \quad$ Fraction of haplotype $T$ which reproduces sexually

$Y \quad$ Fraction of haplotype $t$ which reproduces sexually

$Z$ Cost of sexual reproduction, such that the fitness of sexually reproducing individuals is $(1-Z)$

$p \quad$ Frequency of haplotype $T$ in the current generation

$p^{\prime} \quad$ Frequency of haplotype $T$ in the next generation

$R_{T} \quad$ The rate of growth of the frequenc of $T$ haplotypes, i.e. $p^{\prime} / p$

$R_{t} \quad$ The rate of growth of the frequency of $t$ haplotype, i.e. $\left(1-p^{\prime}\right) /(1-p)$ 
Let $p$ be the population frequency of $T$ in the $n$th generation. The frequency $p^{\prime}$ in the next generation can then be computed as follows. The fraction of the population undergoing sexual reproduction is.

$$
\begin{aligned}
& X \cdot(1-p) \text { from genotype } t, \text { and } \\
& Y \cdot p \text { from genotype } T .
\end{aligned}
$$

The frequencies of $t, T t$ and $T T$ zygotes are then

$$
\frac{X^{2} \cdot(1-p)^{2}}{[X \cdot(1-P)+Y \cdot p]^{2}}, \frac{2 \cdot X \cdot Y \cdot(1-p) \cdot p}{[X \cdot(1-p)+Y \cdot p]^{2}} \text { and } \frac{Y^{2} \cdot p^{2}}{[X \cdot(1-p)+Y \cdot p]^{2}} \text {. }
$$

With respect to the entire population (of sexually and asexually reproducing individuals), the fractional numbers of zygotes of each kind are, for $t t, T t$ and $T T$, respectively

$$
\frac{X^{2} \cdot(1-p)^{2}}{X \cdot(1-p)+Y \cdot p}, \frac{2 \cdot X \cdot Y \cdot(1-p) \cdot p}{X \cdot(1-p)+Y \cdot p} \text { and } \frac{Y^{2} \cdot p^{2}}{X \cdot(1-p)+Y \cdot p} .
$$

Now we can work out the contributions to the next generation from each of the two haplotypes and each mode of reproduction (see figure 1). Denoting the quantity $X \cdot(1-p)+Y \cdot p$ by $C 0$, this goes as follows:

(i) Asexual reproduction of $t$,

$$
C 1=(1-p) \cdot(1 ; X) \text {. }
$$

(ii) Sexual reproduction of $t$,

$$
C 2=(1-p)^{2} \cdot X^{2} \cdot(1-Z) / C 0 .
$$

(iii) Sexual reproduction of untransformed $T t$ zygotes leading to $t$,

$$
C 3=p \cdot(1-p) \cdot X \cdot Y \cdot(1-Z) \cdot(1-F) / C 0 .
$$

(iv) Asexual reproduction of $T$,

$$
C 4=p \cdot(1-Y) \cdot(1-S)
$$

(v) Sexual reproduction of TT zygotes formed by mating,

$$
C 5=p^{2} \cdot Y^{2} \cdot(1-Z) \cdot(1-S) / C 0 .
$$

(vi) Sexual reproduction of untransformed $T t$ leading to $T$,

$$
C 6=p \cdot(1-p) \cdot X \cdot Y \cdot(1-Z) \cdot(1-S) \cdot(1-F) / C 0 .
$$

(vii) Sexual reproduction from $T t$ transformed to $T T$,

$$
C 7=2 \cdot p \cdot(1-p) \cdot X \cdot Y \cdot(1-Z) \cdot(1-S) \cdot F / C 0 .
$$

Finally, $p^{\prime}$, the frequency of $T$ in the next generation is given by the equation

$$
p^{\prime}=\frac{C 4+C 5+C 6+C 7}{C 1+C 2+C 3+C 4+C 5+C 6+C 7} .
$$




\section{Results}

The outcome of the competition between $T$ and $t$ can be visualized from figure 2 , which depicts $p^{\prime}$ (the frequency of $T$ in the next generation) as a function of $p$ (the frequency of $T$ in the current generation) based on (1). If, as in figure $2 \mathrm{a}, p^{\prime}$ is always greater than $p$ (i.e. the curve is always above the line $p=p^{\prime}$ ), $T$ will always increase in frequency and the TEs will go to fixation. Similarly, if the curve is entirely below the line (figure $2 b$ ), $T$ will always be eliminated from the population.

Condition for equilibrium, i.e. $p=p^{\prime}$ (say $=p^{*}$ ) leads to a cubic equation in $p^{*}$, as can be derived from (1). Since $p^{*}=0$ and $p^{*}=1$ always satisfy the condition, there can be at most one more solution with $0<p^{*}<1$; therefore the curve describing $p^{\prime}$ as a function of $p$ can cut the line $p^{\prime}=p$ at just one point between 0 and 1 . If the equilibrium is a stable one (figure $2 \mathrm{c}$ ), there will be a stable polymorphism between $T$ and $t$. If the equilibrium is unstable (figure $2 \mathrm{~d}$ ), the outcome will depend on the initial frequencies of $t$ and $T$. Since $p^{\prime}$ is a monotonic function of $p$ [as can be shown from (1)], there can be no cyclic or chaotic behaviour in this system.

Depending on the values of the parameters of the model viz. $X, Y, Z, S$ and $F$, one of the four outcomes described above is obtained. To arrive at the sets of parameter values which lead to each of these outcomes, the outcome of competition between $T$ and $t$ is explored under two scenarios. In the first, the population is assumed to consist almost entirely of $t$, and its stability with respect to an invasion by $T$ is investigated. The population can be invaded by $T$ if, as $p \rightarrow 0$, the ratio $p^{\prime} / p$ is greater than unity.
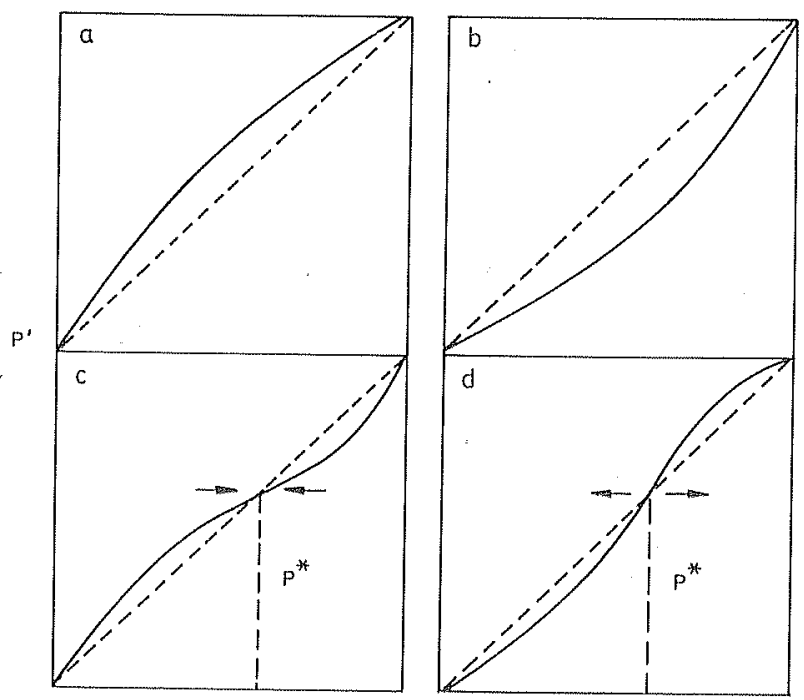

$P$

Figure 2. The four theoretically possible outcomes of the competition between $T$ and $t$ as seen from the schematic plots of $p^{\prime}$ as a function of $p$ (continuous line). (a) $T$ goes to fixation; (b) $t$ goes to fixation; (c) stable polymorphism between $t$ and $T$, (d) initial condition dependence such that if initial $p$ is greater than $p^{*}$, then $T$ goes to fixation; alse $t$ goes to fixation. The broken line corresponds to $p=p^{\prime}$. 
Using $C 1 \ldots C 6$ and (1), this condition can be shown to be

$$
R_{T}=\frac{(1-S) \cdot[1-Y \cdot Z+F \cdot Y \cdot(1-Z)]}{1-X \cdot Z}>1
$$

In the second scenario, the stability of a pure $T$ population is investigated with respect to invasion by $t$. The invasion by $t$ is successful if the ratio $\left(1-p^{\prime}\right) /(1-p)$ is greater than unity as $p \rightarrow 1$ i.e.

$$
R_{t}=\frac{1-Z \cdot X-X \cdot F \cdot(1-Z)}{(1-S) \cdot(1-Y \cdot Z)}>1
$$

The different possible outcomes are

(i) $R_{T}>1$ and $R_{t}<1, T$ goes to fixation.

(ii) $R_{T}<1$ and $R_{t}<1$, initial frequency dependence; both the pure populations stable against invasion.

(iii) $R_{T}<1$ and $R_{t}>1, t$ goes to fixation, and finally

(iv) $R_{T}>1$ and $R_{t}>1$, polymorphism between $T$ and $t$.

Since the five parameters of the model are restricted to the range $0-1$, the entire phase space consists of a five-dimensional hypercube, and the conditions on $R_{T}$ and $R_{t}$ divide this hypercube into regions corresponding to the four outcomes.

Any change in the parameter values which increases $R_{T}$ and decreases $R_{t}$ can be said to favour the $T$ haplotype (transposable elements). An examination of (2) and (3) reveals that:

(i) Low values of $S$ (the selective disadvantage of $T$ ) favour the spread of $T$ as expected.

(ii) High values of $F$ (the transposition frequency) favour $T$.

(iii) High values of $X$ (the proportion of sexually reproducing $t$ individuals) increase $R_{T}$ and decrease $R_{t}$ and thus favour $T$. This is because higher values of $X$ make more $t$ genomes available for $T$ to take over by transposition in the $T t$ diploid zygotes. (iv) A high value of $Y$ (the proportion of sexually reproducing $T$ ) increases $R_{t}$ and makes a $T$ population more susceptible to invasion by $t$. This is because a higher proportion of $T$ has to incur the cost of sexual reproduction.

(v) For both $R_{T}$ and $R_{t}$, the sign of the parameter $Z$ denoting the cost of sexual reproduction is negative both in the numerator and the denominator. The dependence of the outcome on $Z$ is therefore not immediately apparent.

Some special cases, restricting one or more parameters to specific values, are described below.

\subsection{Sexual population: $X=Y=1$}

This corresponds to a situation where, regardless of the presence of a TE, the population does not have an asexual mode of reproduction. The expressions (2) and (3), under these conditions, lead to (after some algebra):

$S<F /(1+F)$, for $T$ to be able to invade $t$ and

$S>F$, for $t$ to be able to invade $T$. 


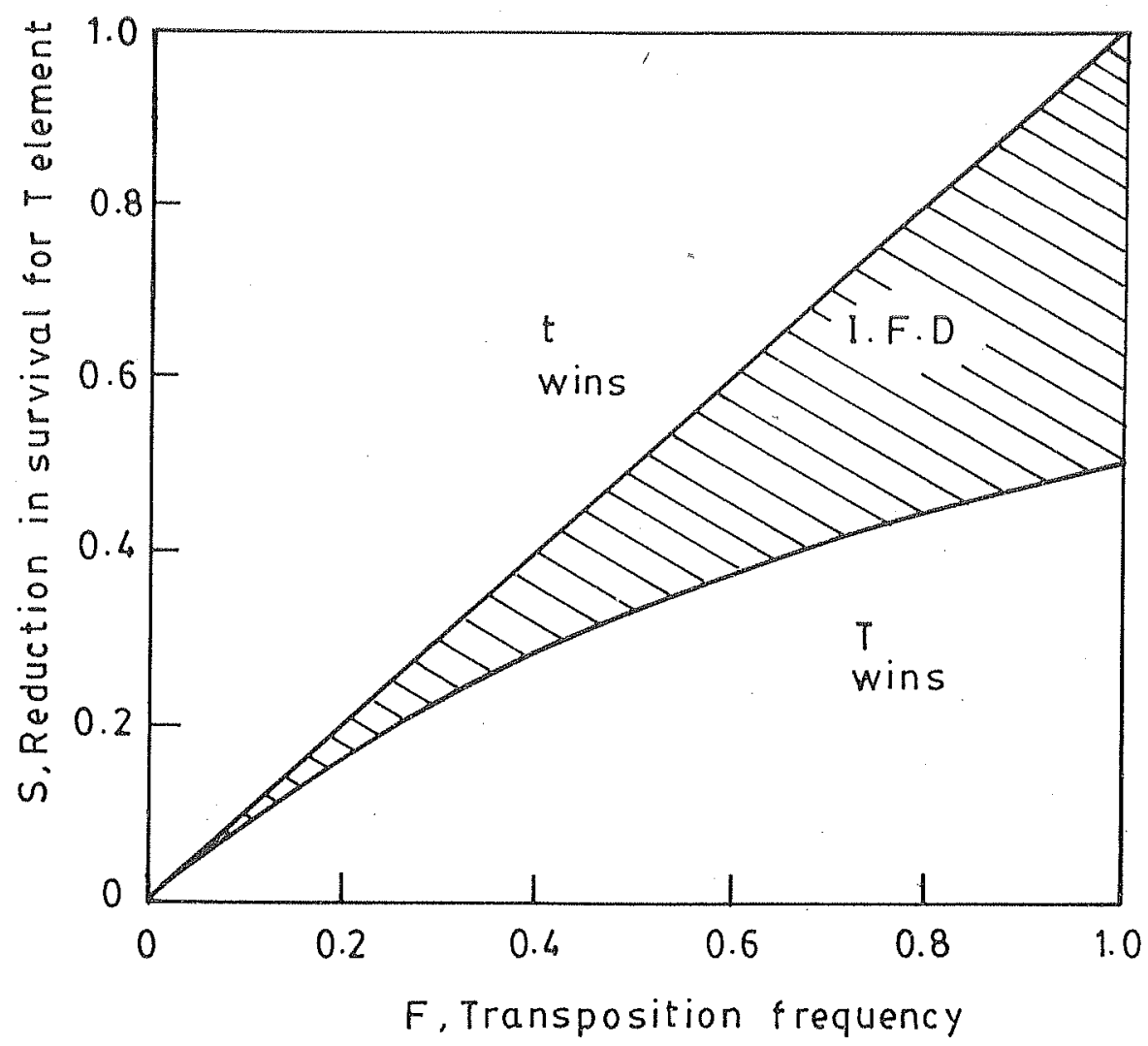

Figure 3. Regions corresponding to different possible outcomes of the competition between $T$ and $t$ in the parameter space of $F$, the transposition frequency and $S$, the selective disadvantage to the TEs. The host population is purely sexual $(X=Y=1)$. Hatched regions correspond to initial frequency dependent outcome (IFD) in this and subsequent figures.

Figure 3 illustrates the possible outcomes of the competition in the $S-F$ space. For $F=1$, the condition for the spread of $T$ is $S<\frac{1}{2}$, as obtained by Hickey (1982). High values of $F$ and low values of $S$ favour $T$ as expected. Interestingly, no combination of $S$ and $F$ can lead to a polymorphism.

Following Nanjundiah (1985), one can explore the effect of the number of copies of $T$ in a diploid zygote on its survival. In particular, $S_{1}$ and $S_{2}$ can be used to denote the survival of the diploid zygote having respectively one or two copies of the TE. If a single copy is assumed to have no adverse effect on survival, a pure $t$ population becomes more susceptible to invasion by $T$, and for a suitable combination of values of $F$ and $S_{2}$, a stable polymorphism between $t$ and $T$ is obtained. This result is consistent with that of Nanjundiah (1985).

\subsection{No effect on sexuality: $X=Y$}

A population with both sexual (fraction $X)$ and asexual $(1-X)$ modes of reproduction is considered, where these proportions are not altered by the presence or absence of TE. Under these conditions, a pure population of $t$ can be invaded by the transposable 


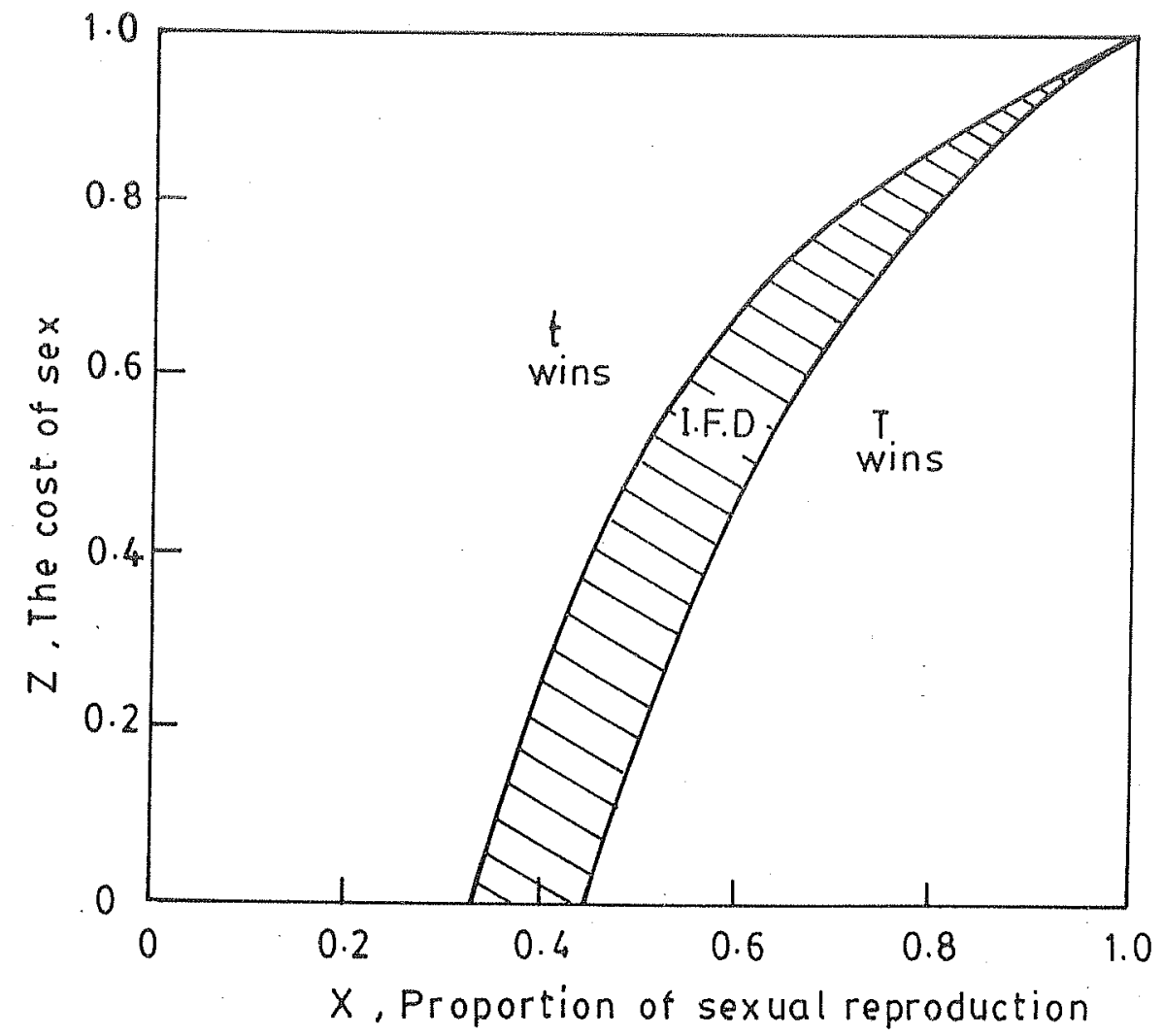

Figure 4. Regions corresponding to different possible outcomes of the competition between $T$ and $t$ in the parameter space of $X$, the proportion of sexual reproduction and $Z$, the cost of sexual reproduction relative to asexual reproduction. The proportion of sexual reproduction is independent of the presence of $T(X=Y)$. Values of the other parameters are: $F=0.75$ and $S=0.25$.

element if

$$
Z<\frac{F \cdot(1-S)-S / X}{F \cdot(1-S)-S}, \text { for } S<F /(1+F)
$$

However, for $S>F /(1+F)$, $t$ is stable in the entire $(Z-X)$ plane. Similarly, a pure population of $T$ is unstable if

$$
Z>\frac{(F-S / X)}{(F-S)}
$$

As shown in figure 4 , high $Z$ (cost of sex) promotes $t$ while high $X$ (proportion of reproduction that is sexual) promotes $T$, as explained earlier. Here, too, a stable polymorphism is not possible.

\subsection{Evolution of sexuality: $Y=1$}

This corresponds to a situation where a host population has both the modes of reproduction, whereas the presence of TE makes sexual reproduction obligatory. A 


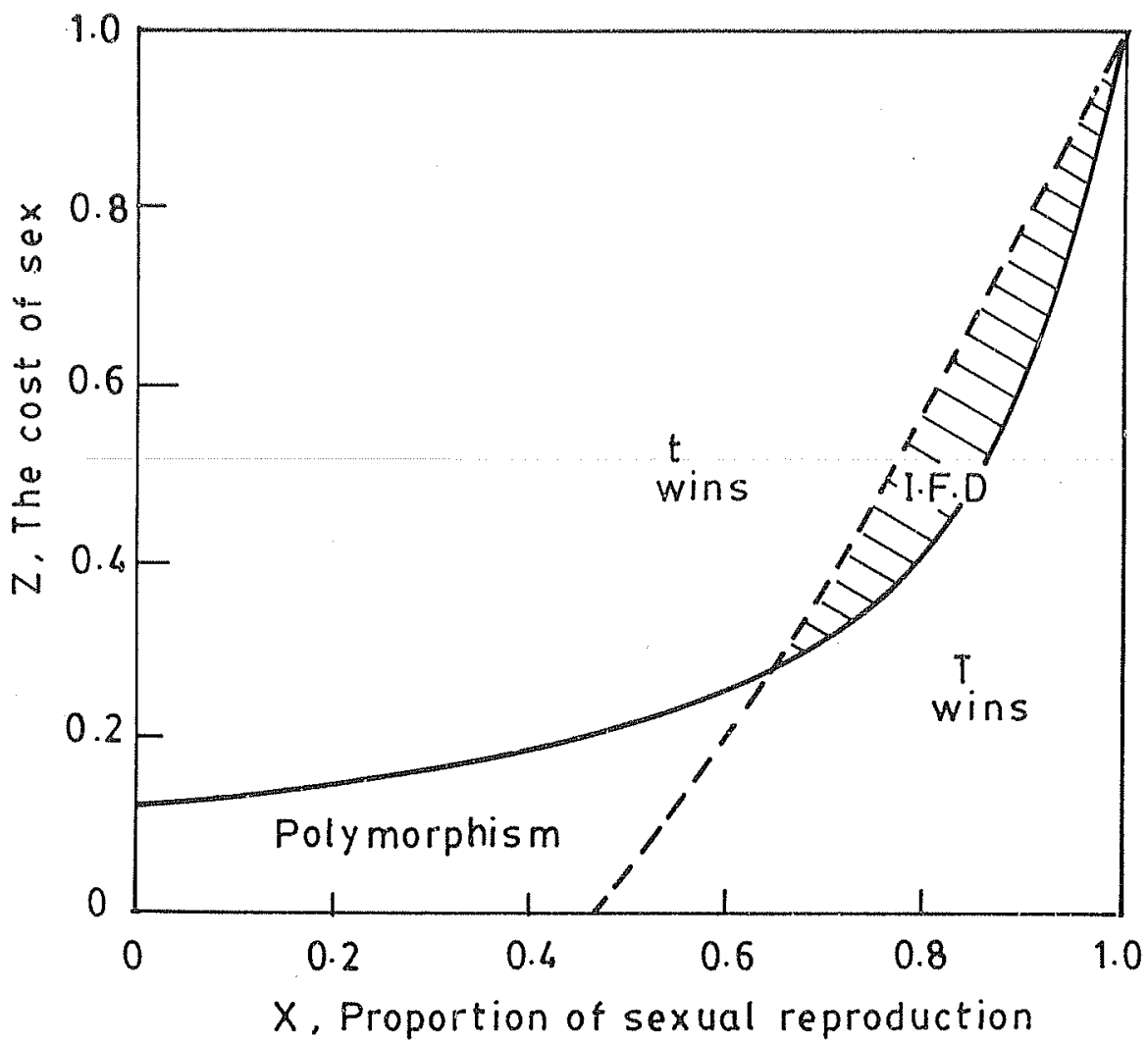

Figure 5. Same as figure 4, except that the presence of transposable elements renders the host purely sexual $(Y=1)$. Values of the other parameters are: $F=0.75$ and $S=0.35$.

pure $t$ population is invadable by $T$ if

$$
Z<\frac{(1-S) \cdot(1+F)-1}{(1-S) \cdot(1+F)-X}
$$

provided $S<F /(1+F)$; otherwise, $t$ is stable in the entire range of combinations of $Z$ and $X$. Similarly, $T$ is invadable by $t$ if

$$
Z>\frac{F \cdot X-S}{(1-S)-X \cdot(1-F)} .
$$

As seen from figure 5, all the four possible outcomes mentioned earlier, i.e. $T$ winning, $t$ winning, initial frequency dependence, and stable polymorphism, can be realized, depending on the values of $X$ and $Z$. As before, high $Z$ favours $t$ and high $X$ favours $T$.

To reiterate, in the region corresponding to high $X$ and low $Z$, a population of $t$ individuals capable of reproducing both sexually and asexually $(0<X<1)$, has been taken over by $T$, which reproduces exclusively sexually $(Y=1)$. The model thus demonstrates the feasibility of transposable element-mediated evolution of sexuality. 


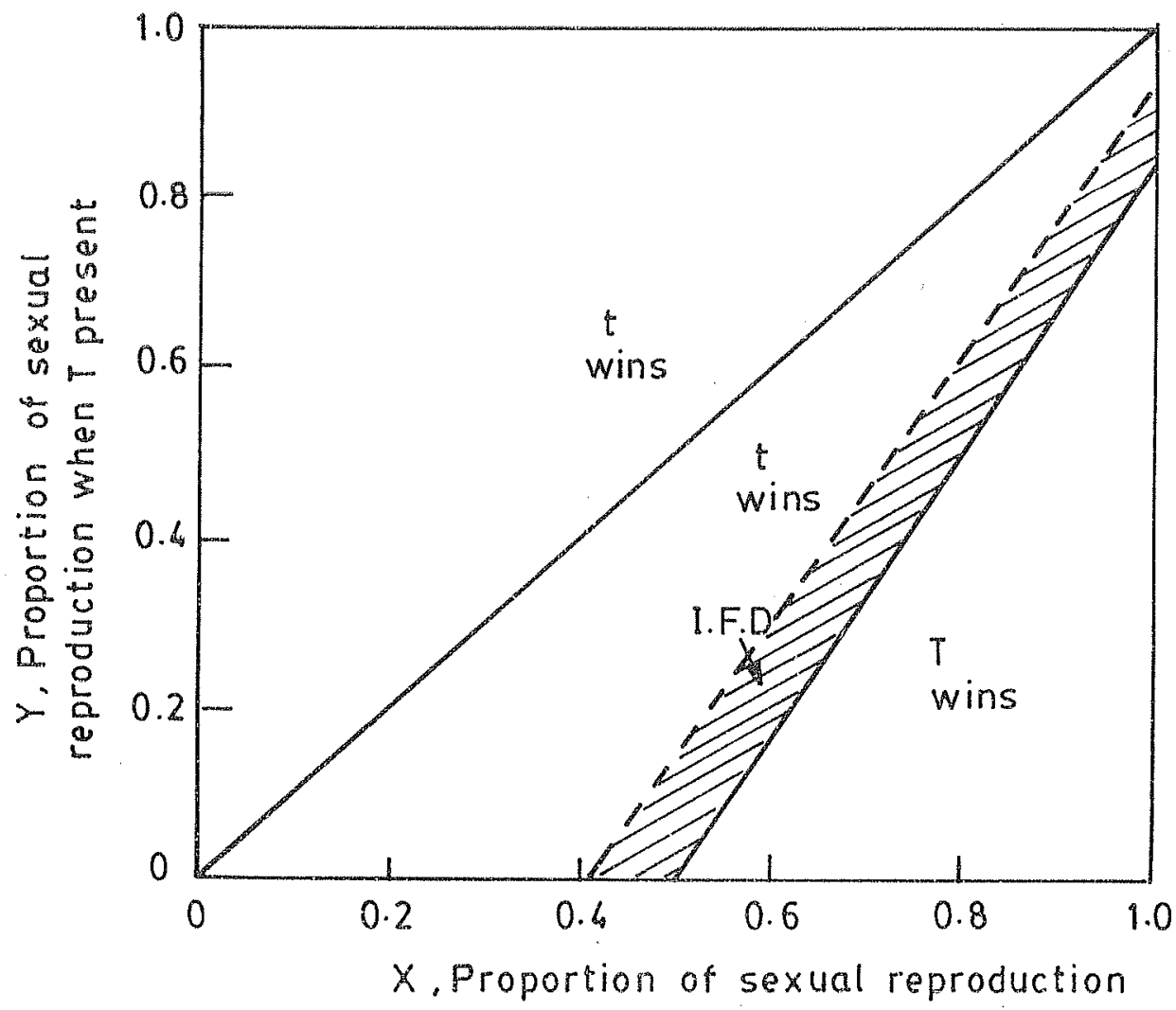

Figure 6. The outcome of competition between $T$ and $t$ in the parameter space of $X$, the proportion of sexual reproduction in the absence of TEs and $Y$, that corresponding to the presence of TEs. Values of the other parameters are: $F=0.20, S=0.25$ and $Z=0.50$.

\subsection{Suppression of sexuality by TES: $Y<X$}

The outcome of competition between $t$ and $T$ is shown in figure 6 in the $(Y-X)$ space, to indicate the effect of different levels of modulation of sexuality by $T$. The figure shows that for a suitable combination of parameters (corresponding to the bottom right corner of the diagram), $T$ can go to fixation for $Y<X$. If a high cost of sexual reproduction is coupled to a low rate of transposition, then a TE which reduces the proportion of sexual reproduction is selected for. In other words, a suppression of sexuality by transposable elements has also been shown to be possible in this model: These arguments can be quantitatively expressed by the condition for the invasion. of $t$ by $T$

$$
Y<\frac{S-X \cdot Z}{(1-S) \cdot[F \cdot(1-Z)-Z]},
$$

if $Z>F /(1+F)$, and the condition for invasion of $T$ by $t$

$$
Y>\frac{X \cdot(Z+F-F \cdot Z)-S}{Z \cdot(1-S)}
$$




\section{Discussion}

The models described here consider a population which has both sexual as well as asexual modes of reproduction, the proportion of individuals adopting one or the other mode being modulated by the presence of TEs. The formulation developed here is also applicable to organisms (such as rotifers) in which several cycles of asexual reproduction are followed by one or more cycles of sexual reproduction. The presence of TEs can then modulate the number of generations of asexual reproduction between two episodes of sexual reproduction.

It may be noted, as pointed out by Hickey (1982) and Nanjundiah (1985), that the model can also be used to describe the evolution of outbreeding. This is due to the formal similarity between inbreeding versus outbreeding on one hand and asexual versus sexual modes of reproduction on the other.

What is the experimental evidence supporting the model? Perhaps the most unambiguous demonstration of transposable elements affecting modes of reproduction is seen in yeast (Klar et al. 1981, see Hickey and Rose 1988), where the switch between the mating types $a$ and $\alpha$ is mediated by the transposable elements $H M L_{\alpha}$ and $H M R_{a}$ (see for example, Zubay 1987). In higher organisms, it is difficult to find such clear-cut examples of TEs influencing sexuality. However, several instances are known of mobile genetic elements producing large phenotypic effects related to sexuality (Green 1980; Werren et al. 1988). Thus, the $P$ elements in Drosophila exhibit hybrid dysgenesis when $P$ males mate with $M$ females but not vice versa. In the fly Megasilia scalaris (Green 1980), the maleness-determining genetic element $M$ is known to be mobile, capable of transposing to nonhomologous chromosomes. Transposable elements are also known to be capable of moving adjacent stretches of DNA along with them during the process of transposition, and short stretches of DNA (in the form of mobile elements or otherwise) can profoundly influence the sexual phenotype. Perhaps one of the most interesting examples of the role played by short stretches of DNA in sex determination is seen in Caenorhabditis elegans (McCoubrey et al. 1988), where an increased copy number of an octanucleotide seems to promote feminization of chromosomal males. Sucli a regulatory function could be performed by the stretch of DNA by serving as a binding site for a regulatory protein (Chandra 1985). In summary, assuming TEs to be capable of influencing the regulatory switch between sexual and asexual reproductive pathways seems quite plausible. It may be worth mentioning that the recent experiments of Stouthamer et al. (1990) have demonstrated that certain microorganisms are capable of converting the parasitic wasp Trichogramma from a sexual to an asexual mode of reproduction.

The ability of TEs to transpose endows them with a considerable fitness advantage. This can offset other costs incurred by them indirectly by way of a lowered fitness of the host. For example, as modelled here, the cost of sex may be imposed on the host by the TEs by inducing sexuality in hosts which are otherwise mostly asexual. On the other hand, sexuality itself is essential for the spread of TEs. The two properties (abinity to tratsspose and sexuality) are thus capable of positively reinforcing each orher. Estimates of the rates of transposition in Drosophila (Charlesworth and Langley 1989) indicate rather low values - of the order of $10^{-4}$ per generation. These are probably too small for the present model to be effective if the cost of sex is substantial. However, these rates are known to increase by an order of magnitude in a suitable genetic background (Good and Hickey 1986) and still higher values do not seem unlikely (Green 1980). 
The maximum rate of spread of TEs is obtained when the transposition frequency is unity, and when the host incurs no cost by harbouring TEs. The fitness advantage in this case is $\frac{1}{2}$, just adequate to counterbalance the two-fold cost of sex. It is therefore unlikely that TEs by themselves would be responsible for the evolution of sexuality. However, the cost of sex may be lowered when one or more of the several processes favouring sexuality (e.g. variable environment, hostile coevolution in host-parasite systems etc.; see Stearns 1990) are operative. Thus, acting in conjunction with these processes, modulation of sexuality by the transposable elements is seen to be a very effective mechanism for the evolution of sex.

\section{Acknowledgements}

This investigation originated and evolved out of many discussions with Drs H. Sharat Chandra and Vidyanand Nanjundiah, who also critically commented on several earlier versions of the manuscript. I also thank Drs Raghavendra Gadagkar, R. Umashankar and K. N. Ganeshiah for many helpful suggestions. A part of this work was presented at the Conference on Sex Determination and Dosage Compensation held at Bangalore, India, in November 1989. Financial assistance from the Department of Environment, Government of India, is gratefully acknowledged.

\section{References}

Bell G. 1982 The masterpiece of nature: The evolution and genetics of sexuality (Berkeley: University of California Press)

Berg D. E. and Howe M. M. (eds) 1989 Mobile DNA (Washington, DC: Am. Soc. Microbiol.)

Bremermann H. J. 1985 The adaptive significance of sexuality. Experientia 41: 1245-1254

Chandra H. S. 1985 Sex determination: A hypothesis based on noncoding DNA. Proc. Natl. Acad. Sci. USA 82: $1165-1169$

Charlesworth B. 1987 The population biology of transposable elements. Trends Ecol. Evol. 2: 21-23

Charlesworth B. 1989 The evolution of sex and recombination. Trends Ecol. Evol. 4: 264-267

Charlesworth B. and Charlesworth D. 1983 The population dynamics of transposable elements. Genet. Res. 42: $1-27$

Charlesworth B. and Langley C. H. 1989 The population genetics of Drosophila transposable elements. Annu. Rev. Genet. 23: 251-287

Doolittle W. F. 1982 Selfish DNA after fourteen months. In Genome evolution (eds) G. A. Dover and R. B. Flavell (London: Academic Press)

Doolittle W. F. and Sapienza C. 1980 Selfish genes, the phenotype paradigm and genome evolution. Nature (London) 284; 601-603

Good A. and Hickey D. A. 1986 The spread of transposable $P$ elements in mixed $P-M$ populations of Drosophila melanogaster. Genetics 113: 72

Green M. M. 1980 Transposable elements in Drosophila and other diptera. Anmu. Rev. Genet. 14: 109-120

Hickey D. A. 1982 Selfish DNA: a sexually transmitted nuclear parasite. Genetics 101: 519-531

Hickey D. A. and Rose M. R. 1988 The role of gene transfer in the evolution of eukaryotic sex. In The evolution of sex (eds) R. E. Michod and B. R. Levin (Sunderland, Mass: Sinaeur Associates)

Hoekstra R. F. 1990 The evolution of male female dimorphism: Older than sex? J. Genet. 69: 11-15

Klar A. J. S., Strathern J. N., Broach J. R. and Hicks J. B. 1981 Regulation of transcription in expressed and unexpressed mating type cassettes of yeast. Nature (London) 289: 239-244

Krieher M. and Rose M. R. 1986 Males, parthenogenesis and the maintenance of anisogamous sex. $J$. Theor. Biol. 122: $421-440$

Langley C. H., Brookfield J. F. Y. and Kaplan N. L. 1983 Transposable elements in Mendelian populations. I. A theory. Genetics 104: 457-472 
Maynard Smith J. 1978 The evolttion of sex (Cambridge: University Press)

McCoubrey W. K., Nordstrom K. D. and Meneely P. M. 1988 Microinjected DNA from the X chromosome affects sex determination in Caenorhabditis elegans. Science 242: 1146-1151

Michod R. and Levin B. R. (eds) 1988 The evolution of sex (Sunderland, Mass: Sinaeur Associates)

Nanjundiah V. 1985 Transposable element copy number and stable polymorphism. J. Genet. 64: 127-134

Ohta T. 1981 Population genetics of selfish DNA. Nature (London) 292: 648-649

Ohta T. 1983 Theoretical study on the accumulation of selfish DNA. Genet. Res. 41: 1-16

Ohta T. and Kimura M. 1981 Some calculations on the amount of sellish DNA. Proc. Natl. Acad. Sci. USA 78: 1129-1132

Orgel L. E. and Crick F. H. C. 1980 Selfish DNA: The ultimate parasite. Nature (London) 284: 604-607

Rose M. R. 1983 The contagion mechanism for the origin of sex. J. Theor. Biol. 101: 137-146

Shapiro J. A. (ed.) 1983 Mobile genetic elements (New York: Academic Press)

Stearns S. C. 1985 The evolution of sex and the role of sex in evolution. Experientia 41: 1231-1235

Stearns S. C. 1990 The evolutionary maintenance of sexual reproduction: The solutions proposed for a longstanding problem. J. Genet. 69: 1-10

Stouthamer R., Luck R. F. and Hamilton W. D. 1990 Antibiotics cause parthenogenetic Trichogramma (Hymenoptera/Trichorammatidac) to revert to sex. Proc. Natl. Acad. Sci. USA 87: 2424-2427

Tremblay C. and Rose M. R. 1985 Population dynamics of gene transfer. Theor. Popul. Biol. 28: 359-381

Werren J. H., Nur U. and Wu Chung I. 1988 Selfish genetic elements. Trends Ecol. Evol. 3: 297--302

Williams G. C. 1975 Sex and evolution: (Monographs in population biology) (Princeton: University Press)

Zinder N. 1985 The origin of sex: An argument. In The origin and evolution of sex (eds) H. O. Halvorson and A. Monroy (New York: Alan R. Liss)

Zubay G. 1987 Genetics (Menlo Park, CA: Benjamin/Cummings) 